\title{
Association of fried food consumption with all cause, cardiovascular, and cancer mortality: prospective cohort study
}

\author{
Yangbo Sun, ${ }^{1}$ Buyun Liu, ${ }^{1}$ Linda G Snetselaar, ${ }^{1}$ Jennifer G Robinson, ${ }^{1,2}$ Robert B Wallace, ${ }^{1}$ \\ Lindsay L Peterson, ${ }^{3}$ Wei Bao ${ }^{1,4,5}$
}

Check for updates

${ }^{1}$ Department of Epidemiology,

College of Public Health,

University of lowa, lowa City,

IA, USA

${ }^{2}$ Department of Internal Medicine, Carver College of

Medicine, University of lowa, lowa City, IA, USA

${ }^{3}$ Division of Medical Oncology, Department of Medicine,

Washington University School of Medicine, St Louis, MO, USA

${ }^{4}$ Obesity Research and Education Initiative, University

of lowa, lowa City, IA, USA

${ }^{5}$ Fraternal Order of Eagles

Diabetes Research Center,

University of lowa, lowa City,

IA, USA

Correspondence to: W Bao,

Department of Epidemiology,

College of Public Health,

University of lowa, $145 \mathrm{~N}$

Riverside Drive, Room 5431

CPHB, lowa City, IA 52242, USA

wei-bao@uiowa.edu

(ORCID 0000-0002-7301-5786)

Additional material is published online only. To view please visit the journal online.

Cite this as: $B M J$ 2019;364:5420 http://dx.doi.org/10.1136/bmj.5420

Accepted: 17 December 2018

\section{ABSTRACT}

OBJECTIVE

To examine the prospective association of total and individual fried food consumption with all cause and cause specific mortality in women in the United States.

DESIGN

Prospective cohort study.

SETTING

Women's Health Initiative conducted in 40 clinical centers in the US.

\section{PARTICIPANTS}

106966 postmenopausal women aged $50-79$ at study entry who were enrolled between September 1993 and 1998 in the Women's Health Initiative and followed until February 2017.

\section{MAIN OUTCOME MEASURES}

All cause mortality, cardiovascular mortality, and cancer mortality.

\section{RESULTS}

31558 deaths occurred during 1914691 person years of follow-up. For total fried food consumption, when comparing at least one serving per day with no consumption, the multivariable adjusted hazard ratio was 1.08 (95\% confidence interval 1.01 to 1.16) for all cause mortality and 1.08 (0.96 to 1.22) for cardiovascular mortality. When comparing at least one serving per week of fried chicken with no consumption, the hazard ratio was 1.13 (1.07 to 1.19) for all cause mortality and 1.12 (1.02 to 1.23 ) for cardiovascular mortality. For fried fish/shellfish, the corresponding hazard ratios were 1.07 (1.03 to 1.12) for all cause mortality and 1.13 (1.04 to 1.22 ) for cardiovascular mortality. Total or individual fried food consumption was not generally associated with cancer mortality.

\section{CONCLUSIONS}

Frequent consumption of fried foods, especially fried chicken and fried fish/shellfish, was associated with a higher risk of all cause and cardiovascular mortality in women in the US.

\section{WHAT IS ALREADY KNOWN ON THIS TOPIC}

Around $25-36 \%$ of North American adults consume foods, usually fried, from fast food restaurants every day

Scientific evidence about the effect of fried foods on mortality is sparse and controversial

\section{WHAT THIS STUDY ADDS}

Fried food consumption, especially fried chicken and fried fish/shellfish, was observed to be associated with a higher risk of all cause and cardiovascular mortality among postmenopausal women in the United States

\section{Introduction}

Fried foods are widely consumed in the United States and worldwide. Frying is a complex cooking process that modifies the composition of foods and the frying medium through oxidation, polymerization, and hydrogenation. ${ }^{1}$ During frying, foods can lose water and absorb fat, and the frying oils deteriorate, especially when reused. ${ }^{1}$ Moreover, frying makes food crunchy and more appetizing, which can lead to excess intake. $^{2}$

Several cohort studies in US populations showed that higher consumption of fried foods was associated with an increased risk of type 2 diabetes $^{3}$ and cardiovascular diseases, ${ }^{34}$ which are among the leading causes of death. However, a study in a Mediterranean population found no association between fried food consumption and coronary heart disease. ${ }^{1}$ Little is known about the relation between fried food consumption and mortality. ${ }^{15}$ It is important to understand the associations between fried foods and health outcomes because $25-36 \%$ of North American adults consume foods, usually fried, from fast food restaurants every day. $^{367}$ Thus, we used data from a large, prospective cohort to examine the association of total and specific fried food consumption with all cause, cardiovascular, and cancer mortality in US women.

\section{Methods \\ Study population}

The Women's Health Initiative has been previously described in detail. ${ }^{8}$ Briefly, between 1993 and 1998, postmenopausal women aged 50-79 at study entry were recruited through 40 clinical centers. Most clinics used a tracking system to calculate the response rate to their mailings and other recruitment efforts, but the types of system and information tracked varied widely across centers. No study wide system was implemented because the clinics wanted to maintain as much local flexibility as possible. Therefore, the response rate (that is, the number of women making contact for initial screening) varied across clinics and sources of mailing lists from less than $2 \%$ to about $20 \%$ for initial mailings; however, total mailing numbers and response rates to mailings are not available across clinics. A total of 373092 women completed the initial screening form. Of these women, 68132 (18\%) underwent the subsequent screening visits to be randomized into the clinical trial, and 93676 (25\%) were enrolled in the observational study. The clinical trial consisted of four components: a dietary modification trial, two hormonal treatment trials, and a calcium and vitamin D clinical trial. The clinical trial and observational study were closed in 2004-05 and the participants 
were invited to continue being followed in the Women's Health Initiative extension study, which has follow-up data to February 2017. Written informed consent was obtained from each participant. Institutional review board approval was obtained from all participating institutions.

In this study, we included participants in the observational study component and clinical trial components, with the exception of the dietary modification trial. This is because participants in the dietary modification trial were randomized to evaluate the effects of a low fat diet, ${ }^{9}$ which could affect their habitual consumption of fried foods. Of the eligible participants, 108308 women (90009 (96\%) in the observational study; 18299 (95\%) in the clinical trial) had valid food frequency questionnaire data, which were defined as reported energy intake between 600 and $5000 \mathrm{kcal} / \mathrm{day}$ (1 kcal=4.18 kJ=0.00418 MJ). We excluded 104 women who had missing data on information about postmenopausal hormone therapy use. We also excluded 1238 women who died within three years after the baseline visit, to rule out the possibility that the participants changed their diet because of their preterminal or terminal illness. Therefore, 106966 women (88881 in the observational study; 18085 in the clinical trial) were included in the present analysis.

\section{Dietary assessment}

We used a standardized written protocol, centralized training of staff, and quality assurance visits by the clinical coordinating center to ensure uniform administration of data collection. Diet was measured at baseline in the Women's Health Initiative using a self administered food frequency questionnaire developed and validated with characteristics described for the study, ${ }^{10}$ adapted from the health habits and lifestyle questionnaire. ${ }^{11}$ The three sections of the Women's Health Initiative's food frequency questionnaire included 122 composite and single food line items asking about frequency of consumption and portion size, and 19 adjustment questions related to type of fat intake. There were also four summary questions asking about the usual intake of fruits and vegetables and added fats for comparison with information gathered from the line items. The questionnaire was designed to record food intake relevant to multiethnic and geographically diverse population groups. The questionnaire has been shown to produce reliable estimates (correlation coefficient $r_{\text {all nutrients }}=0.76$ ) that are comparable to those for eight days of dietary intake compiled from four 24 hour dietary recalls and four days of food records $(r=0.37,0.62,0.41,0.36$ for energy, percentage of energy from fat, carbohydrate, and protein, respectively). ${ }^{10}$ The nutrient database used to analyze the questionnaire is derived from the nutrition data systems for research, ${ }^{12}$ which provides nutrient information for more than 140 nutrients and compounds, including energy, saturated fat, and sodium.

Total fried food consumption was the total consumption of the following three items: fried chicken, fried fish/shellfish, and other fried foods. Fried chicken was described as "fried chicken" on the questionnaire. Fried fish/shellfish was described as "fried fish, fish sandwich, and fried shellfish (shrimp and oysters)" on the questionnaire. Other questionnaire items on fried foods included "French fries, fried potatoes, fried rice, fried cassava and fritters," "snacks such as potato chips, corn chips, tortilla chips, pork skins, Ritz and cheese crackers," "fried plantains," "taco and tostada," "flauta and crispy rolled taco," and "Indian fried bread" on the questionnaire. We assumed that all these categories consisted primarily of deep fried foods. ${ }^{13}$ There were exceptions, such as "Ritz and cheese crackers" as part of the snacks category. However, the contribution of these single items within their general category is likely to be small. ${ }^{13}$ The primary exposure of this study was total fried food consumption, and the secondary exposures were individual types of fried foods.

\section{Ascertainment of death}

Deaths were ascertained by reviewing death certificates, medical records, autopsy reports, and by linkage to the national death index. ${ }^{14}$ Death certificates and hospital records were obtained and adjudicated by adjudicators who were unaware of study components or randomization assignment. Deaths in the clinical trial component of the Women's Health Initiative were centrally adjudicated, as were major causes of cardiovascular death and the five main cancer outcomes. Other deaths were adjudicated locally. ${ }^{14}$ Records from the most relevant hospital admission preceding death and from the time of death, autopsy records, and the death certificate were used by adjudicators to determine the causes of death. For many deaths occurring out of hospital, documentation was limited to the death certificate and records of the most recent admission to hospital before death. In these instances, the immediate and underlying cause of death was determined from the death certificate. ${ }^{14}$ Ascertainment of outcomes was complete as of 28 February 2017. Mortality endpoints for this study included all cause (primary outcome), cardiovascular, and cancer mortality.

\section{Other covariate assessments}

The following information was collected at baseline through self reporting: demographic characteristics (age, race/ethnicity, education, annual income), lifestyle (smoking status, physical activity, alcohol intake, coffee intake, total energy intake, overall diet quality), medical history (cardiovascular disease, cancer, diabetes), and drug use and past hormone use (unopposed estrogen use, estrogen plus progesterone use). Overall diet quality was indicated by alternative healthy eating index 2010 score. ${ }^{15}$ This score was based on the intake levels of 11 components: vegetables, fruits, whole grains, sugar-sweetened beverages and fruit juice, nuts and legumes, red and processed meat, trans fat, long chain (n-3) fats (eicosapentaenoic and docosahexaenoic acid), polyunsaturated fatty 
acids, sodium, and alcohol. Recreational moderate to vigorous intensity physical activity, including walking, was assessed by questionnaire, and metabolic equivalent task hours per week of physical activity for each participant was calculated, as described in detail elsewhere. ${ }^{816}$ Weight and height at baseline were measured during clinic visits using standard methods. We calculated body mass index as weight (in kilograms) divided by height (in meters squared). ${ }^{2}$

\section{Statistical analysis}

Comparisons of covariates among different groups were performed using analysis of variance for continuous variables and $\chi^{2}$ test for categorical variables. We used Cox proportional hazards models to estimate age adjusted and multivariable adjusted hazard ratios and 95\% confidence intervals for mortality associated with total fried food consumption and its components. Person years were calculated from the baseline food frequency questionnaire until death, the last national death index search date, or the end of the previously described Women's Health Initiative extension study 2 on 28 February 2017. Model 1 adjusted for the following variables: age at baseline, race or ethnicity, education, annual income, observational study or clinical trial, unopposed estrogen use, estrogen plus progesterone use, smoking status, physical activity, coffee intake, total energy intake, alternative healthy eating index 2010 score, baseline diabetes, cardiovascular disease, and cancer. For each of the three fried food items, mutual adjustment for other fried food items was also added. Model 2 additionally adjusted for body mass index, which was a potential mediator of the study association reported in the literature. ${ }^{1}$

We evaluated whether the associations would vary according to the following variables: age $(<65 v \geq 65)$, race or ethnicity (white $v$ non-white), smoking status (never smoked $v$ ever smoked), physical activity $(<10$ $v \geq 10$ metabolic equivalent task hours per week), unopposed estrogen use (never used $v$ ever used), estrogen plus progesterone use (never used $v$ ever used), oil used for frying at home (no fat added, olive or canola oil, others (other oils, butter, margarine, etc) or mixed use of oils), and obesity status (body mass index $<30 v \geq 30$ ). For sensitivity analysis, we repeated the analyses by excluding women in the trials, adding types of oil used for home frying into the model, and adding trans fatty acid intake into the model.

All statistical tests were based on a priori hypotheses and therefore there was no adjustment for multiple testing. All statistical analyses were conducted using SAS (version 9.4; SAS Institute). All tests were two sided with statistical significance set at $\mathrm{P}<0.05$.

\section{Patient and public involvement}

No patients were involved in setting the research question or the outcome measures, nor were they involved in developing plans for recruitment, design, or implementation of the study. No patients were asked to advise on interpretation or writing up of results. This study used deidentified information collected in a national health study. There are no plans to disseminate the results of the research to study participants or the relevant patient community.

\section{Results}

During 1914691 person years of follow-up (individual level mean follow-up duration 17.9 years), 31558 deaths occurred, consisting of 9320 deaths from cardiovascular disease, 8358 deaths from cancer, and 13880 deaths from other causes. Table 1 shows that women with more frequent total fried food consumption were more likely to be younger, non-white, with less education, and on lower income. They were more likely to be participants from the Women's Health Initiative clinical trials, be current smokers, have lower physical activity levels, drink more coffee, have a higher total energy intake, and have a lower diet quality. They were also more likely to have diabetes but less likely to have cardiovascular disease at baseline, and more likely to have a higher body mass index. Women with higher total fried food consumption were more likely to consume calories from dietary total fat rather than carbohydrate or total protein. They tended to consume fewer vegetables, fruits, and whole grains, and more sugar-sweetened beverages, nuts and legumes, red and processed meat, trans fat, polyunsaturated fatty acid, and sodium. There was a moderate correlation between two of the three fried food items: fried chicken and fried fish/shellfish; $r=0.40, \mathrm{P}<0.001$.

More frequent total fried food consumption was associated with a higher risk of all cause mortality (table 2): multivariable adjusted hazard ratio 1.01 (95\% confidence interval 0.98 to 1.05 ) for less than one serving per week, 1.03 (0.99 to 1.07) for one to two servings per week, 1.03 (0.99 to 1.08) for three to six servings per week, and 1.08 (1.01 to 1.16) for at least one serving per day ( $\mathrm{P}=0.02$ for trend) compared with no consumption. For the specific fried food items, fried chicken consumption was associated with a higher risk of all cause mortality: 1.06 (1.03 to 1.09) for less than two servings per month, 1.12 (1.07 to 1.17$)$ for two to three servings per month, and 1.13 (1.07 to 1.19) for at least one serving per week $(\mathrm{P}<0.001$ for trend) compared with no consumption (table 3 ). Fried fish/ shellfish consumption of at least one serving per week was associated with a higher risk of all cause mortality $(1.07 ; 1.03$ to 1.12$)$. Other fried food consumption was not associated with all cause mortality. These associations persisted after additional adjustment for body mass index.

Total fried food consumption of at least one serving per day was associated with a modestly higher but not significant risk of cardiovascular mortality: hazard ratio 1.08 (95\% confidence interval 0.96 to 1.22 ; table 2). Fried chicken consumption was associated with a higher risk of cardiovascular mortality: 1.08 (1.02 to 1.14 ) for less than two servings per month, 1.17 (1.08 to 1.25) for two to three servings per month, and 1.12 (1.02 to 1.23) for at least one serving per week $(\mathrm{P}<0.001$ for trend) compared with no consumption (table 4). Fried fish/shellfish consumption of at least 


\section{Table 1 | Demographic characteristics according to frequency of baseline total fried food consumption. Data are percentages or mean (standard} deviation) unless indicated otherwise

\begin{tabular}{|c|c|c|c|c|c|c|}
\hline \multirow[b]{2}{*}{ Variables } & \multicolumn{5}{|c|}{ Frequency of total fried food consumption } & \multirow[b]{2}{*}{ P } \\
\hline & None $(n=15166)$ & $\begin{array}{l}<1 \text { serving/week } \\
(n=38482)\end{array}$ & $\begin{array}{l}1-2 \text { servings/week } \\
(n=33210)\end{array}$ & $\begin{array}{l}3-6 \text { servings/week } \\
(n=15480)\end{array}$ & $\begin{array}{l}\geq 1 \text { serving/day } \\
(\mathrm{n}=4628)\end{array}$ & \\
\hline Age at baseline (years) & $64.9(7.3)$ & $64.3(7.3)$ & $63.1(7.3)$ & $62.4(7.2)$ & $61.3(7.3)$ & $<0.001$ \\
\hline \multicolumn{7}{|l|}{ Race/ethnicity: } \\
\hline White & 90.5 & 87.9 & 82.2 & 77.1 & 68.5 & \multirow[t]{5}{*}{$<0.001$} \\
\hline Black & 4.4 & 5.5 & 8.5 & 11.3 & 17.2 & \\
\hline Hispanic & 2.4 & 3.0 & 4.2 & 5.7 & 7.7 & \\
\hline Others & 2.5 & 3.3 & 5.0 & 5.8 & 6.5 & \\
\hline Missing & 0.3 & 0.3 & 0.2 & 0.2 & 0.2 & \\
\hline \multicolumn{7}{|l|}{ Education: } \\
\hline High school or less & 24.6 & 28.8 & 34.2 & 38.8 & 44.9 & \multirow[t]{5}{*}{$<0.001$} \\
\hline Some college & 25.2 & 27.0 & 27.2 & 26.9 & 25.4 & \\
\hline College & 12.8 & 11.7 & 10.7 & 9.9 & 8.8 & \\
\hline Postgraduate & 36.7 & 31.8 & 27.2 & 23.4 & 19.9 & \\
\hline Missing & 0.7 & 0.8 & 0.8 & 0.9 & 1.0 & \\
\hline \multicolumn{7}{|l|}{ Annual income: } \\
\hline$<20000$ & 13.6 & 14.5 & 15.5 & 18.2 & 21.6 & \multirow[t]{4}{*}{$<0.001$} \\
\hline $20000-49999$ & 38.1 & 40.7 & 42.7 & 42.4 & 43.1 & \\
\hline$>50000$ & 40.5 & 38.0 & 35.0 & 32.4 & 28.1 & \\
\hline Missing & 7.9 & 6.8 & 6.8 & 6.9 & 7.3 & \\
\hline \multicolumn{7}{|l|}{ WHI component: } \\
\hline Clinical trial & 13.7 & 15.7 & 17.9 & 19.8 & 20.6 & \multirow[t]{2}{*}{$<0.001$} \\
\hline Observational study & 86.3 & 84.3 & 82.2 & 80.2 & 79.4 & \\
\hline \multicolumn{7}{|l|}{ Unopposed estrogen use: } \\
\hline Never used & 67.1 & 64.5 & 64.0 & 65.9 & 66.8 & \multirow[t]{3}{*}{$<0.001$} \\
\hline Past user & 13.0 & 13.7 & 13.6 & 13.1 & 12.8 & \\
\hline Current user & 20.0 & 21.8 & 22.4 & 21.0 & 20.4 & \\
\hline Estrogen+progesterone use: & & & & & & \\
\hline Never used & 73.4 & 72.7 & 74.4 & 76.1 & 78.9 & $<0.001$ \\
\hline Past user & 8.6 & 9.1 & 8.7 & 8.7 & 6.7 & \\
\hline Current user & 18.0 & 18.2 & 16.9 & 15.3 & 14.5 & \\
\hline Smoking status: & & & & & & \\
\hline Never & 48.9 & 50.2 & 50.6 & 49.6 & 47.8 & $<0.001$ \\
\hline Past & 45.7 & 42.7 & 40.4 & 39.7 & 40.3 & \\
\hline Current & 4.0 & 5.6 & 7.6 & 9.5 & 10.8 & \\
\hline Missing & 1.3 & 1.4 & 1.4 & 1.3 & 1.2 & \\
\hline Physical activity (MET-h/week): & & & & & & \\
\hline$<10$ & 36.3 & 45.2 & 53.5 & 59.8 & 64.7 & $<0.001$ \\
\hline$\geq 10$ & 61.9 & 52.8 & 44.2 & 38.0 & 33.1 & \\
\hline Missing & 1.9 & 2.0 & 2.3 & 2.2 & 2.3 & \\
\hline Alcohol intake: & & & & & & \\
\hline Non-drinker & 42.0 & 38.8 & 39.6 & 42.7 & 48.3 & $<0.001$ \\
\hline Moderate & 45.1 & 47.8 & 46.9 & 43.5 & 39.6 & \\
\hline Heavy & 12.9 & 12.4 & 13.4 & 13.7 & 12.1 & \\
\hline Coffee intake (medium serving/day) & $2.1(1.7)$ & $2.0(1.7)$ & $2.1(1.8)$ & $2.2(1.9)$ & $2.4(2.2)$ & $<0.001$ \\
\hline Total energy intake (kcal/day) ${ }^{*}$ & $1377.1(470.6)$ & $1401.4(485.6)$ & $1593.7(551.0)$ & $1906.2(644.3)$ & $2470.4(834.7)$ & $<0.001$ \\
\hline AHEI 2010 score & $55.7(10.6)$ & $51.1(10.5)$ & $47.1(10.2)$ & $44.4(10.3)$ & $42.7(10.4)$ & $<0.001$ \\
\hline Baseline diabetes & 4.5 & 4.9 & 5.8 & 7.3 & 9.7 & $<0.001$ \\
\hline Baseline cardiovascular disease & 7.0 & 6.3 & 6.3 & 5.8 & 6.1 & $<0.001$ \\
\hline Baseline cancer & 1.5 & 1.5 & 1.5 & 1.6 & 1.3 & 0.73 \\
\hline Body mass index: & & & & & & \\
\hline$<25.0$ & 49.6 & 43.3 & 35.3 & 29.2 & 23.9 & $<0.001$ \\
\hline $25.0-29.9$ & 31.9 & 34.4 & 34.8 & 34.0 & 30.6 & \\
\hline$\geq 30$ & 17.3 & 21.2 & 29.0 & 35.8 & 44.3 & \\
\hline Missing & 1.2 & 1.1 & 1.0 & 1.0 & 1.3 & \\
\hline Dietary carbohydrate (\%E) & $58.7(9.6)$ & $54.5(9.1)$ & $50.2(8.6)$ & $47.2(8.3)$ & $45.0(8.0)$ & $<0.001$ \\
\hline Dietary total fat (\%E) & $23.6(7.5)$ & $27.9(7.5)$ & $32.5(7.4)$ & $36.0(7.3)$ & $39.0(7.0)$ & $<0.001$ \\
\hline Dietary total protein (\%E) & $17.8(3.6)$ & $17.1(3.3)$ & $16.7(3.1)$ & $16.2(3.0)$ & $15.7(3.1)$ & $<0.001$ \\
\hline Vegetables (servings/day) & $2.9(1.5)$ & $2.5(1.4)$ & $2.3(1.3)$ & $2.3(1.3)$ & $2.5(1.4)$ & $<0.001$ \\
\hline Fruits (servings/day) & $3.1(1.9)$ & $2.5(1.8)$ & $2.1(1.7)$ & $1.9(1.6)$ & $1.7(1.6)$ & $<0.001$ \\
\hline Whole grains (g/day) & $41.0(31.9)$ & $35.0(28.0)$ & $32.9(27.5)$ & $32.8(27.9)$ & $35.0(30.0)$ & $<0.001$ \\
\hline SSBs (servings/day) & $0.7(0.8)$ & $0.8(0.8)$ & $0.9(0.9)$ & $1.0(1.0)$ & $1.2(1.4)$ & $<0.001$ \\
\hline Nuts and legumes (servings/day) & $0.2(0.3)$ & $0.2(0.3)$ & $0.2(0.3)$ & $0.3(0.3)$ & $0.4(0.4)$ & $<0.001$ \\
\hline Red and processed meat (servings/day) & $0.7(0.7)$ & $0.9(0.8)$ & $1.3(0.9)$ & $1.7(1.2)$ & $2.3(1.6)$ & $<0.001$ \\
\hline Trans fat (\%E) & $1.4(0.9)$ & $1.8(1.0)$ & $2.3(1.0)$ & $2.7(1.1)$ & $3.0(1.0)$ & $<0.001$ \\
\hline EPA+DHA (mg/day) & $141.2(151.2)$ & $115.6(117.9)$ & $118.7(106.2)$ & $136.1(126.7)$ & $175.8(200.1)$ & $<0.001$ \\
\hline
\end{tabular}




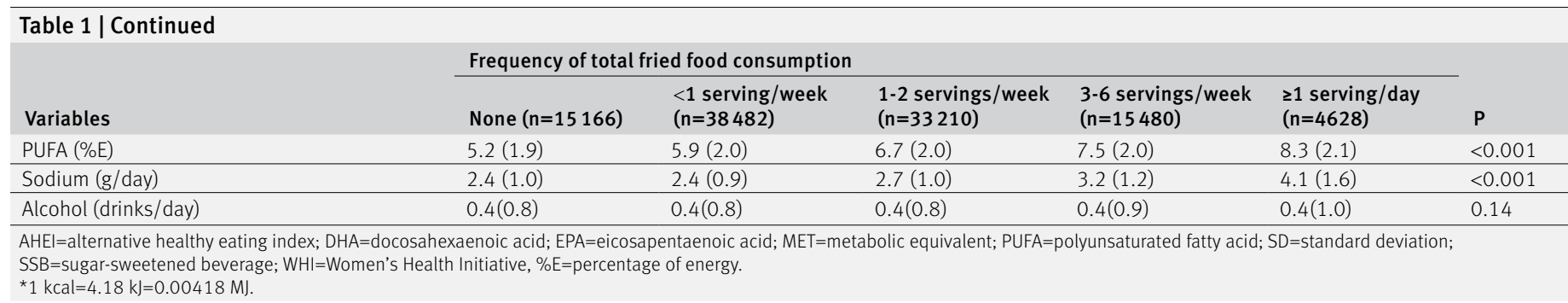

one serving per week was associated with a higher risk for cardiovascular mortality: 1.13 (1.04 to 1.22; table 4). Other fried foods were generally not associated with cardiovascular mortality. These associations persisted after additional adjustment for body mass index.

Neither total nor specific fried food consumption was associated with cancer mortality (table 2 and table 5). However, for fried fish/shellfish consumption of less than two servings per month, the hazard ratio was 0.92 (95\% confidence interval 0.87 to 0.97 ), and for other fried food consumption of less than two servings per month, the hazard ratio was 1.09 (1.02 to 1.17) compared with no consumption (table 5). These associations persisted after adjusting for body mass index.

The results of sensitivity analyses were similar when women in the clinical trial were excluded, oils used for frying were added into the model, or trans fatty acid intake was added into the model (eTables 2-3). The association of total or specific fried food consumption with all cause mortality did not vary by race or ethnicity, physical activity, or oil used for frying ( $P$ values $\geq 0.06$ ). The association of total or specific fried food consumption with all cause mortality generally tended to be more obvious among women younger than 65, those who ever smoked, those who never used unopposed estrogen or estrogen plus progesterone, and those without obesity (eTable 4). The association of total or specific fried food consumption with cardiovascular mortality did not vary by race or ethnicity, smoking status, physical activity, unopposed estrogen use, estrogen plus progesterone use, oil used for frying, or obesity status ( $P$ values $\geq 0.07$ ). The association of fried chicken or fried fish/shellfish consumption with cardiovascular mortality was more obvious among women younger than 65 (eTable 5). Generally, the association of total or specific fried food consumption with cancer mortality did not vary by age, race/ethnicity, smoking status, physical activity, unopposed estrogen use, or oil used for frying ( $P$ values $\geq 0.08$ ); in addition, the association did not vary by obesity status or estrogen plus progesterone use after stratification (eTable 6).

\section{Discussion}

In this large prospective cohort study, we comprehensively examined the associations of total and specific fried foods with all cause, cardiovascular, and cancer mortality. We found a significantly positive association of fried food consumption, especially fried chicken and fried fish/shellfish, with risk of all cause and cardiovascular mortality. These associations were slightly attenuated, but remained significant, after additional adjustment for a variety of factors that were related to mortality, including age, race/ethnicity, socioeconomic status, hormone use, lifestyle factors, health status, and body mass index.

\section{Results in relation to other studies}

Previous studies on fried food consumption and mortality are scarce ${ }^{15}$ and their findings have been

\begin{tabular}{|c|c|c|c|c|c|c|}
\hline \multirow[b]{2}{*}{ Mortality } & \multicolumn{5}{|c|}{ Frequency of total fried food consumption } & \multirow[b]{2}{*}{$P$ for trend } \\
\hline & None & $<1$ serving/week & $1-2$ servings/week & $3-6$ servings/week & $\geq 1$ serving/day & \\
\hline \multicolumn{7}{|l|}{ All cause mortality: } \\
\hline No of deaths/participants & $4693 / 15166$ & $11755 / 38482$ & $9529 / 33210$ & $4283 / 15480$ & $1298 / 4628$ & 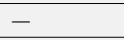 \\
\hline Age adjusted model & 1.00 (ref) & $1.06(1.02$ to 1.09$)$ & $1.13(1.10$ to 1.17$)$ & $1.20(1.15$ to 1.25$)$ & $1.36(1.28$ to 1.44$)$ & $<0.001$ \\
\hline Multivariable model 1 & 1.00 (ref) & $1.01(0.98$ to 1.05$)$ & 1.03 (0.99 to 1.07$)$ & 1.03 (0.99 to 1.08$)$ & $1.08(1.01$ to 1.16$)$ & 0.02 \\
\hline Multivariable model 2 & 1.00 (ref) & $1.01(0.98$ to 1.05$)$ & $1.02(0.99$ to 1.06$)$ & $1.03(0.98$ to 1.07$)$ & 1.07 (1.01 to 1.15$)$ & 0.06 \\
\hline \multicolumn{7}{|l|}{ Cardiovascular mortality: } \\
\hline No of deaths/participants & $1416 / 15166$ & $3456 / 38482$ & $2842 / 33210$ & $1221 / 15480$ & $385 / 4628$ & - \\
\hline Age adjusted model & 1.00 (ref) & $1.04(0.98$ to 1.11$)$ & $1.16(1.09$ to 1.24$)$ & 1.19 (1.10 to 1.29$)$ & $1.43(1.27$ to 1.60$)$ & $<0.001$ \\
\hline Multivariable model 1 & 1.00 (ref) & $1.00(0.94$ to 1.06$)$ & 1.03 (0.97 to 1.10$)$ & $1.00(0.92$ to 1.09$)$ & 1.08 (0.96 to 1.22$)$ & 0.29 \\
\hline Multivariable model 2 & 1.00 (ref) & $0.99(0.93$ to 1.06$)$ & 1.02 (0.95 to 1.09$)$ & $0.99(0.91$ to 1.07$)$ & 1.06 (0.94 to 1.20$)$ & 0.57 \\
\hline \multicolumn{7}{|l|}{ Cancer mortality: } \\
\hline No of deaths/participants & $1180 / 15166$ & $3071 / 38482$ & $2568 / 33210$ & $1203 / 15480$ & $336 / 4628$ & - \\
\hline Age adjusted model & 1.00 (ref) & $1.06(0.99$ to 1.14$)$ & $1.11(1.03$ to 1.18$)$ & $1.16(1.07$ to 1.26$)$ & $1.16(1.02$ to 1.31$)$ & $<0.001$ \\
\hline Multivariable model 1 & 1.00 (ref) & $1.03(0.96$ to 1.10$)$ & $1.02(0.95$ to 1.09$)$ & $1.01(0.93$ to 1.10$)$ & 0.95 (0.83 to 1.08$)$ & 0.61 \\
\hline Multivariable model 2 & 1.00 (ref) & 1.03 (0.96 to 1.10$)$ & 1.01 (0.94 to 1.08$)$ & $1.00(0.92$ to 1.09$)$ & $0.93(0.82$ to 1.06$)$ & 0.41 \\
\hline
\end{tabular}




\begin{tabular}{|c|c|c|c|c|c|}
\hline \multirow[b]{2}{*}{ Fried food items } & \multicolumn{4}{|c|}{ Frequency of consumption } & \multirow[b]{2}{*}{ P for trend } \\
\hline & None & $<2$ servings/month & $2-3$ servings/month & $\geq 1$ serving/week & \\
\hline \multicolumn{6}{|l|}{ Fried chicken: } \\
\hline No of deaths/participants & $19788 / 68709$ & $6642 / 21980$ & $3188 / 10268$ & $1940 / 6009$ & - \\
\hline Age adjusted model & 1.00 (ref) & $1.14(1.11$ to 1.17$)$ & $1.26(1.22$ to 1.31$)$ & $1.38(1.31$ to 1.44$)$ & $<0.001$ \\
\hline Multivariable model 1 & 1.00 (ref) & 1.06 (1.03 to 1.09$)$ & $1.12(1.07$ to 1.17$)$ & $1.13(1.07$ to 1.19$)$ & $<0.001$ \\
\hline Multivariable model 2 & 1.00 (ref) & 1.06 (1.03 to 1.09$)$ & 1.11 (1.07 to 1.16$)$ & $1.12(1.06$ to 1.18$)$ & $<0.001$ \\
\hline \multicolumn{6}{|l|}{ Fried fish/shellfish: } \\
\hline No of deaths/participants & $17549 / 60169$ & $7292 / 24958$ & $4017 / 13489$ & $2700 / 8350$ & - \\
\hline Age adjusted model & 1.00 (ref) & 1.04 (1.01 to 1.07$)$ & $1.11(1.07$ to 1.15$)$ & $1.22(1.17$ to 1.27$)$ & $<0.001$ \\
\hline Multivariable model 1 & 1.00 (ref) & 0.97 (0.94 to 0.99) & $0.99(0.96$ to 1.03$)$ & 1.07 (1.03 to 1.12$)$ & 0.07 \\
\hline Multivariable model 2 & 1.00 (ref) & $0.97(0.94$ to 0.99$)$ & $0.99(0.95$ to 1.03$)$ & $1.07(1.02$ to 1.12$)$ & 0.10 \\
\hline \multicolumn{6}{|l|}{ Other fried foods: } \\
\hline No of deaths/participants & $6613 / 20484$ & $7166 / 22699$ & $5669 / 19468$ & $12110 / 44315$ & - \\
\hline Age adjusted model & 1.00 (ref) & 1.04 (1.01 to 1.08$)$ & 1.04 (1.01 to 1.08$)$ & $1.10(1.07$ to 1.14$)$ & $<0.001$ \\
\hline Multivariable model 1 & 1.00 (ref) & 1.01 (0.98 to 1.05$)$ & 0.98 (0.95 to 1.03$)$ & $0.98(0.95$ to 1.01$)$ & 0.06 \\
\hline Multivariable model 2 & 1.00 (ref) & 1.01 (0.98 to 1.05$)$ & $0.98(0.95$ to 1.02$)$ & $0.98(0.95$ to 1.01$)$ & 0.05 \\
\hline
\end{tabular}

inconsistent. Similar to our findings, a previous study in the US found that fried food consumption was associated with the risk of incident type 2 diabetes and cardiovascular disease. ${ }^{3}$ Additionally, the observed associations of fried chicken consumption and fried fish/shellfish consumption with cardiovascular mortality were consistent with previous studies. These studies found fried food consumption was associated with a higher risk of heart failure, ${ }^{417}$ acute myocardial infarction, ${ }^{18-20}$ total ischemic heart disease, ${ }^{21}$ and death. $^{22}$ We are aware that a previous study in Spain, a Mediterranean country, reported that fried food consumption was not associated with all cause mortality. ${ }^{1}$ Frying practice and medium vary by cooking tradition and the location where fried foods are consumed. For example, in the US, fried foods are eaten more often away from home (eg, in fast food restaurants) than at home; away from home, fried foods are usually deep fried ${ }^{24}$ in corn oil, which is the most common frying medium. ${ }^{25}$ In Mediterranean countries, however, fried foods are consumed at home as frequently as away from home, with pan frying and deep frying commonly used, ${ }^{26}$ and mostly with olive oil for home frying. ${ }^{27} 28$ The types of oil used for frying in Spain (mainly olive oil and sunflower oil) could partially explain the difference observed between our study and the study in Spain. Some studies showed that olive oil consumption was associated with a decreased risk of overall and cardiovascular mortality. ${ }^{29} 30$

Thus it is important to examine the associations of fried foods with mortality in different settings because the results of studies from one setting are not necessarily applicable to another. No previous study has examined the association of fried food consumption with cancer mortality.

\begin{tabular}{|c|c|c|c|c|c|}
\hline \multirow[b]{2}{*}{ Fried food items } & \multicolumn{4}{|c|}{ Frequency of consumption } & \multirow[b]{2}{*}{$P$ for trend } \\
\hline & None & $<2$ servings/month & $2-3$ servings/month & $\geq 1$ serving/week & \\
\hline \multicolumn{6}{|l|}{ Fried chicken: } \\
\hline No of deaths/participants & $5721 / 68709$ & $2005 / 21980$ & $986 / 10268$ & $608 / 6009$ & - \\
\hline Age adjusted model & 1.00 (ref) & 1.19 (1.13 to 1.26$)$ & 1.37 (1.28 to 1.47$)$ & 1.51 (1.39 to 1.64$)$ & $<0.001$ \\
\hline Multivariable model 1 & 1.00 (ref) & $1.08(1.02$ to 1.14$)$ & $1.17(1.08$ to 1.25$)$ & $1.12(1.02$ to 1.23$)$ & $<0.001$ \\
\hline Multivariable model 2 & 1.00 (ref) & 1.07 (1.02 to 1.13$)$ & $1.15(1.07$ to 1.24$)$ & $1.11(1.01$ to 1.21$)$ & $<0.001$ \\
\hline \multicolumn{6}{|l|}{ Fried fish/shellfish: } \\
\hline No of deaths/participants & $5067 / 60169$ & $2209 / 24958$ & $1173 / 13489$ & $871 / 8350$ & - \\
\hline Age adjusted model & 1.00 (ref) & 1.09 (1.04 to 1.15$)$ & $1.14(1.07$ to 1.21$)$ & 1.37 (1.27 to 1.47$)$ & $<0.001$ \\
\hline Multivariable model 1 & 1.00 (ref) & $1.00(0.95$ to 1.06$)$ & $0.98(0.92$ to 1.05$)$ & 1.13 (1.04 to 1.22$)$ & 0.05 \\
\hline Multivariable model 2 & 1.00 (ref) & $1.00(0.95$ to 1.05$)$ & $0.97(0.91$ to 1.04$)$ & 1.12 (1.04 to 1.22$)$ & 0.09 \\
\hline \multicolumn{6}{|l|}{ Other fried foods: } \\
\hline No of deaths/participants & $2041 / 20484$ & $2136 / 22699$ & $1656 / 19468$ & $3487 / 44315$ & - \\
\hline Age adjusted model & 1.00 (ref) & $1.02(0.96$ to 1.08$)$ & $1.02(0.95$ to 1.08$)$ & 1.09 (1.03 to 1.15$)$ & 0.002 \\
\hline Multivariable model 1 & 1.00 (ref) & $0.99(0.93$ to 1.05$)$ & $0.96(0.89$ to 1.02$)$ & 0.95 (0.90 to 1.01) & 0.09 \\
\hline Multivariable model 2 & 1.00 (ref) & 0.98 (0.93 to 1.05$)$ & 0.95 (0.89 to 1.02$)$ & 0.95 (0.89 to 1.01) & 0.06 \\
\hline $\begin{array}{l}\text { Multivariable model } 1=\text { age at bas } \\
\text { trial, unopposed estrogen use, es } \\
\text { baseline diabetes status, baseline } \\
\text { ref=reference. }\end{array}$ & e, race/ethnicity, & cation, annual income, $\mathrm{w}$ & $\begin{array}{l}\text { ther they were from the o } \\
\text { ctivity, coffee intake, tota }\end{array}$ & $\begin{array}{l}\text { servational study or the } \\
\text { energy intake, diet qualit } \\
\text { ivariable model } 1+\text { body }\end{array}$ & $\begin{array}{l}\text { hical } \\
\text { core, } \\
\text { ass index; }\end{array}$ \\
\hline
\end{tabular}




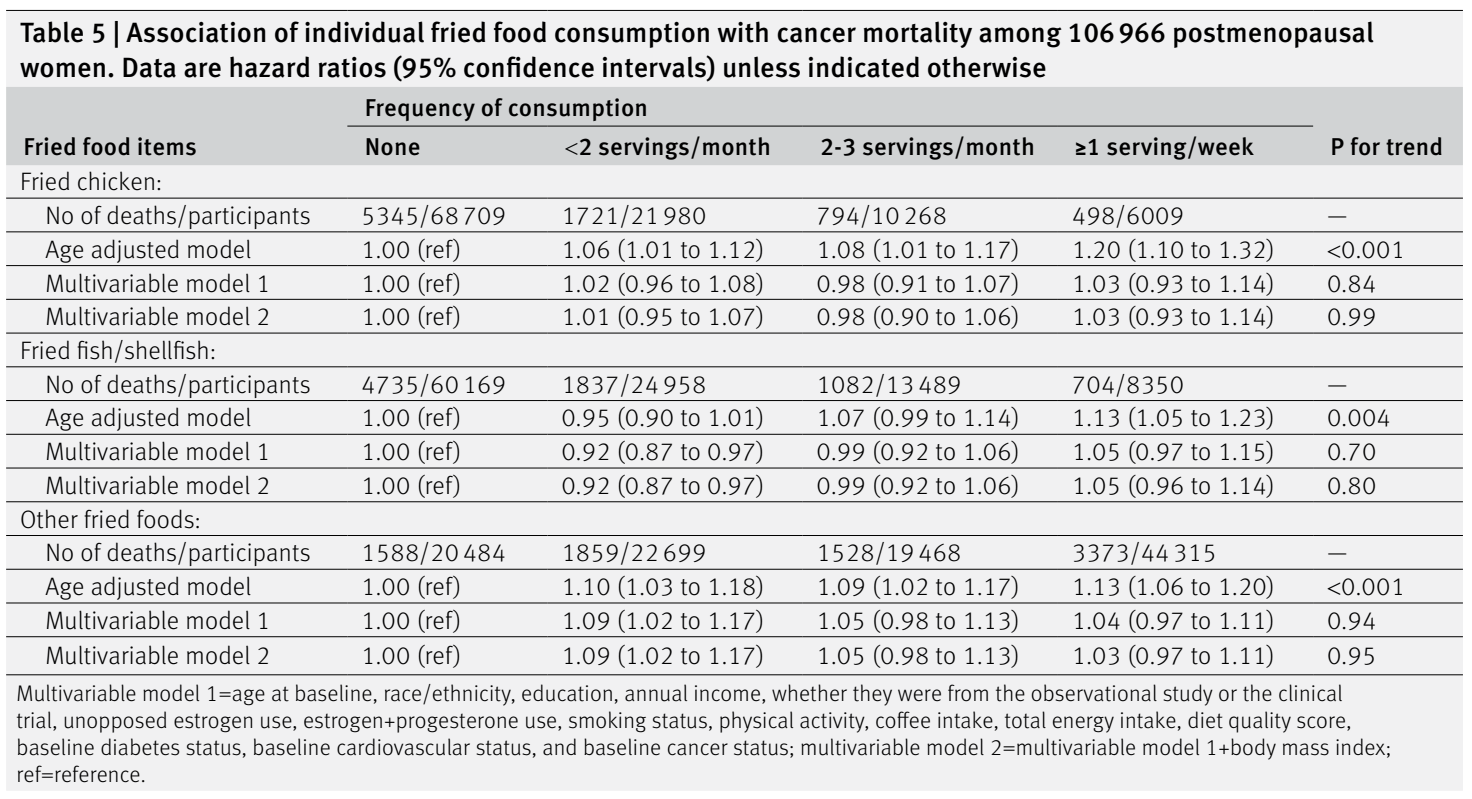

\section{Possible explanations of findings}

There are several possible explanations for the associations of fried foods with cardiovascular and all cause mortality, although the detailed mechanisms are not well understood. The food could lose water and absorb fats during frying, ${ }^{31}{ }^{32}$ which would increase the energy density of food. Frying could also lead to excess energy intake by making food more aromatic and appealing in texture, thus improving food palatability. ${ }^{23}$ Additionally, frying deteriorates oils through the process of oxidation and hydrogenation, leading to a loss of unsaturated fatty acids such as linoleic acid and an increase in the corresponding trans fatty acids such as trans linoleic acid. ${ }^{134}$

In our study, the associations persisted after adjustment for total energy intake and additional adjustment for trans fatty acid intake, suggesting that the associations could not be fully explained by increased energy intake or trans fatty acid. Another possibility is the higher levels of dietary advanced glycation end products as a result of frying, ${ }^{3536}$ which could have an important role in the development of cardiovascular disease, mainly through induction of oxidative stress and inflammation. ${ }^{37-39}$ Previous studies have shown that higher levels of dietary advanced glycation end products were associated with higher risk of all cause and cardiovascular mortality. ${ }^{4041}$ Moreover, frying at a high temperature can lead to acrylamide formation in some foods, such as French fries and potato chips. Dietary acrylamide has been associated with higher risk of all cause, cardiovascular, and cancer mortality. ${ }^{3942}$ One possible explanation for the lack of association between fried foods and cancer mortality in our study could be that the dietary intake of acrylamide from fried foods might not be high enough to have an effect on cancer mortality; it could also be because of the different types of cancers. Further investigation is needed to examine the associations of fried foods with different cancer sites. In addition, reused cooking fat could inhibit the activity of paraoxonase, an enzyme that inhibits the activity of low density lipoprotein cholesterol, ${ }^{43}$ which is involved in the pathogenesis of atherosclerosis. ${ }^{44}$ Finally, some of the fried foods such as fried chicken or fried fish could be ultra-processed foods that are usually high in added sodium, which could partially contribute to the higher risk of mortality related to these foods. ${ }^{45}$

Further studies are warranted to fully understand the mechanisms for the associations of fried foods with all cause and cardiovascular mortality. For example, studies could compare the same foods fried with different frying oils, fried with the same oil at different temperatures, or cooked using different methods such as grilling. These comparisons could help determine the role of different oils, the extent of oil degradation, and the changes in the food itself in the associations of fried foods with all cause and cardiovascular mortality. It is also important to explore the associations of fried foods with different cancer types to better understand the associations of fried foods and cancer mortality.

\section{Strengths and limitations of study}

Our study had several strengths, including the large sample size, the prospective study design which could establish the temporal direction of the associations, and the long term follow-up. Although the Women's Health Initiative was not designed to be a nationally representative sample of US women, the geographical, socioeconomic, and racial or ethnic diversity of the participants could improve the generalizability of the findings to other populations with similar characteristics. We also had detailed data on confounders that could potentially alter the association of fried food consumption with mortality, thus we could explore the role of fried food consumption thoroughly.

We acknowledge that there are several limitations. Firstly, we do not know the degree of browning, 
duration, temperature, and method (deep or pan) used for frying or how often oils were reused. We have information on the oils that were used for frying at home, but we do not know the proportion of fried foods consumed at home or away from home. Therefore, the information provided is limited and it is not possible to determine whether and how the associations between fried foods and mortality differ by the type of oil used for frying. Secondly, we cannot separate the effect of a specific food from the effect of frying for any fried food. For example, we cannot separate the effect of frying from the potentially protective effects of fish/shellfish, which could in part explain the reduced risk of cancer mortality in those consuming some fish versus no fried fish. Thirdly, on the food frequency questionnaire, some fried foods were grouped together as one item. For example, fried potatoes and French fries were not separated from fried rice, fried cassava, and fritters. Therefore, we could not separate the associations of each of these items with mortality in the present study. Previous studies on fried potato consumption and mortality have yielded inconsistent results. ${ }^{1546}$ Finally, as in other observational studies, even though we have adjusted for a wide variety of covariates that relate to mortality, residual confounding by unidentified confounders is still possible.

\section{Conclusions and public health implications}

We have identified a risk factor for cardiovascular mortality that is readily modifiable by lifestyle and cooking choices. Reducing the consumption of fried foods, especially fried chicken and fried fish/shellfish, could have a clinically meaningful effect across the public health spectrum. In conclusion, in this large prospective cohort study, we observed that fried food consumption, especially fried chicken and fried fish/ shellfish, was associated with a higher risk of all cause and cardiovascular mortality among postmenopausal women in the US. Further studies are warranted to elucidate the underlying mechanisms.

We acknowledge the dedicated efforts of investigators and staff at the Women's Health Initiative (WHI) clinical centers, the WHI Clinical Coordinating Center, and the National Heart, Lung, and Blood program office (listing available at www.whi.org). We also recognize the extraordinary commitment of participants to the WHI program. For a list of all the investigators who have contributed to WHI Science, please visit: http://www.whiscience.org/wp-content/uploads/WHI_ investigators_longlist.pdf.

Contributors: YS and WB designed research. YS conducted research, analyzed data, and wrote the paper. All authors contributed to the acquisition, analysis, or interpretation of the data, and revised the manuscript for important intellectual content. WB has primary responsibility for final content and is the study guarantor. All authors read and approved the final manuscript. The corresponding author attests that all listed authors meet authorship criteria and that no others meeting the criteria have been omitted.

Funding: The WHI program is funded by the National Heart, Lung, and Blood Institute, National Institutes of Health, and US Department of Health and Human Services through contracts HHSN268201600018C, HHSN268201600001C HHSN268201600002C, HHSN268201600003C, and HHSN268201600004C. This manuscript was prepared in collaboration with investigators of the WHI, and has been reviewed and approved by the WHI. The funders had no role in the design and conduct of the study, the collection, analysis, and interpretation of the data, or the preparation, review, or approval of the manuscript.

Competing interests: All authors have completed the ICMJE uniform disclosure form at www.icmje.org/coi_disclosure.pdf and declare: no financial relationships with any organisations that might have an interest in the submitted work in the previous three years; no other relationships or activities that could appear to have influenced the submitted work.

Ethical approval: The WHI was overseen by ethics committees at all 40 clinical centers, by the coordinating center, and by a data and safety monitoring board. Each institution obtained human subjects committee approval. Each participant provided written informed consent.

Data sharing: No additional data available.

The lead author affirms that the manuscript is an honest, accurate, and transparent account of the study being reported; that no important aspects of the study have been omitted; and that any discrepancies from the study as planned (and, if relevant, registered) have been explained.

This is an Open Access article distributed in accordance with the Creative Commons Attribution Non Commercial (CC BY-NC 4.0) license, which permits others to distribute, remix, adapt, build upon this work non-commercially, and license their derivative works on different terms, provided the original work is properly cited and the use is noncommercial. See: http://creativecommons.org/licenses/by-nc/4.0/.

1 Guallar-Castillón P, Rodríguez-Artalejo F, Lopez-Garcia E, et al. Consumption of fried foods and risk of coronary heart disease: Spanish cohort of the European Prospective Investigation into Cancer and Nutrition study. BM/ 2012;344:e363. doi:10.1136/bmj.e363

2 Guallar-Castillón P, Rodríguez-Artalejo F, Fornés NS, et al. Intake of fried foods is associated with obesity in the cohort of Spanish adults from the European Prospective Investigation into Cancer and Nutrition. Am J Clin Nutr 2007;86:198-205. doi:10.1093/ ajcn/86.1.198

3 Cahill LE, Pan A, Chiuve SE, et al. Fried-food consumption and risk of type 2 diabetes and coronary artery disease: a prospective study in 2 cohorts of US women and men. Am J Clin Nutr 2014;100:667-75. doi:10.3945/ajcn.114.084129

4 Djoussé L, Petrone AB, Gaziano JM. Consumption of fried foods and risk of heart failure in the physicians' health study. I Am Heart Assoc 2015:4:e001740 doi:10.1161/JAHA.114.001740

5 Veronese N, Stubbs B, Noale M, et al. Fried potato consumption is associated with elevated mortality: an 8-y longitudinal cohort study. Am / Clin Nutr 2017:106:162-7. doi:10.3945/ajcn.117.154872

6 Powell LM, Nguyen BT, Han E. Energy intake from restaurants: demographics and socioeconomics, 2003-2008. Am J Prev Med 2012;43:498-504. doi:10.1016/j.amepre.2012.07.041

7 Morse KL, Driskell JA. Observed sex differences in fast-food consumption and nutrition self-assessments and beliefs of college students. Nutr Res 2009;29:173-9. doi:10.1016/j. nutres 2009.02 .004

8 The Women's Health Initiative Study Group. Design of the Women's Health Initiative clinical trial and observational study. Control Clin Trials 1998:19.61-109. doi:10.1016/S0197-2456(97)00078-0

9 Ritenbaugh C, Patterson RE, Chlebowski RT, et al. The Women's Health Initiative Dietary Modification trial: overview and baseline characteristics of participants. Ann Epidemiol 2003;13(Suppl):S87-97. doi:10.1016/S10472797(03)00044-9

10 Patterson RE, Kristal AR, Tinker LF, Carter RA, Bolton MP, AgursCollins T. Measurement characteristics of the Women's Health Initiative food frequency questionnaire. Ann Epidemiol 1999;9:178 87. doi:10.1016/S1047-2797(98)00055-6

11 Block G, Hartman AM, Dresser CM, Carroll MD, Gannon J, Gardner L. A data-based approach to diet questionnaire design and testing. Am J Epidemiol 1986;124:453-69. doi:10.1093/oxfordjournals.aje. a114416

12 Schakel SF, Sievert YA, Buzzard IM. Sources of data for developing and maintaining a nutrient database. J Am Diet Assoc 1988;88:126871.

13 Osorio-Yáñez C, Gelaye B, Qiu C, et al. Maternal intake of fried foods and risk of gestational diabetes mellitus. Ann Epidemiol 2017;27:384-390.e1. doi:10.1016/j. annepidem.2017.05.006

14 Curb JD, McTiernan A, Heckbert SR, et al, WHI Morbidity and Mortality Committee. Outcomes ascertainment and adjudication methods in the Women's Health Initiative. Ann Epidemiol 2003;13(Suppl):S122-8. doi:10.1016/S10472797(03)00048-6 
15 Chiuve SE, Fung TT, Rimm EB, et al. Alternative dietary indices both strongly predict risk of chronic disease. J Nutr 2012;142:1009-18 doi:10.3945/jn.111.157222

16 Johnson-Kozlow M, Rock CL, Gilpin EA, Hollenbach KA, Pierce JP. Validation of the WHI brief physical activity questionnaire among women diagnosed with breast cancer. Am / Health Behav 2007;31:193-202. doi:10.5993/AJHB.31.2.8

17 Belin RJ, Greenland P, Martin L, et al. Fish intake and the risk of incident heart failure: the Women's Health Initiative. Circ Heart Fail 2011;4:404-13. doi:10.1161/ CIRCHEARTFAILURE.110.960450

18 Iqbal R, Anand S, Ounpuu S, et al, INTERHEART Study Investigators. Dietary patterns and the risk of acute myocardial infarction in 52 countries: results of the INTERHEART study. Circulation 2008;118:1929-37. doi:10.1161/ CIRCULATIONAHA.107.738716

19 Kabagambe EK, Baylin A, Siles X, Campos H. Individual saturated fatty acids and nonfatal acute myocardial infarction in Costa Rica. Eur J Clin Nutr 2003;57:1447-57. doi:10.1038/sj.ejcn.1601709

20 Guo J, Li W, Wang Y, et al, INTERHEART China study investigators. Influence of dietary patterns on the risk of acute myocardial infarction in China population: the INTERHEART China study. Chin Med I (Engl) 2013;126:464-70.

21 Zamora-Ros R, Castañeda J, Rinaldi S, et al. Consumption of fish is not associated with risk of differentiated thyroid carcinoma in the European Prospective Investigation into Cancer and Nutrition (EPIC) Study. J Nutr 2017;147:1366-73. doi:10.3945/jn.117.247874

22 Mozaffarian D, Lemaitre RN, Kuller LH, Burke GL, Tracy RP, Siscovick DS, Cardiovascular Health Study. Cardiac benefits of fish consumption may depend on the type of fish meal consumed the Cardiovascular Health Study. Circulation 2003;107:1372-7. doi:10.1161/01.CIR.0000055315.79177.16

23 Panwar RB, Gupta R, Gupta BK, et al. Atherothrombotic risk factors \& premature coronary heart disease in India: a case-control study. Indian J Med Res 2011;134:26-32.

24 Guthrie JF, Lin BH, Frazao E. Role of food prepared away from home in the American diet, 1977-78 versus 1994-96: changes and consequences. J Nutr Educ Behav 2002;34:140-50. doi:10.1016/ S1499-4046(06)60083-3

25 Jahren AH, Schubert BA. Corn content of French fry oil from national chain vs. small business restaurants. Proc Natl Acad Sci U S A 2010;107:2099-101. doi:10.1073/pnas.0914437107

26 Rohrmann S, Linseisen J, Becker N, et al, European Prospective Investigation into Cancer and Nutrition. Cooking of meat and fish in Europe--results from the European Prospective Investigation into Cancer and Nutrition (EPIC). Eur J Clin Nutr 2002;56:1216-30. doi:10.1038/sj.ejcn.1601494

27 Sayon-Orea C, Bes-Rastrollo M, Basterra-Gortari FJ, et al. Consumption of fried foods and weight gain in a Mediterranean cohort: the SUN project. Nutr Metab Cardiovasc Dis 2013;23:14450. doi:10.1016/j.numecd.2011.03.014

28 Soriguer F, Almaraz MC, García-Almeida JM, et al. Intake and home use of olive oil or mixed oils in relation to healthy lifestyles in a Mediterranean population. Findings from the prospective Pizarra study. Br J Nutr 2010;103:114-22. doi:10.1017/ S0007114509991498

29 Guasch-Ferré M, Hu FB, Martínez-González MA, et al. Olive oil intake and risk of cardiovascular disease and mortality in the PREDIMED Study. BMC Med 2014;12:78. doi:10.1186/17417015-12-78

30 Buckland G, Mayén AL, Agudo A, et al. Olive oil intake and mortality within the Spanish population (EPIC-Spain). Am J Clin Nutr 2012;96:142-9. doi:10.3945/ajcn.111.024216
31 Fillion L, Henry CJ. Nutrient losses and gains during frying: a review. Int J Food Sci Nutr 1998;49:157-68. doi:10.3109/09637489809089395

32 Pokorn J, Pánek J, Trojáková L. Effect of food component changes during frying on the nutrition value of fried food. Forum Nutr 2003:56:348-50.

33 de Souza RJ, Mente A, Maroleanu A, et al. Intake of saturated and trans unsaturated fatty acids and risk of all cause mortality, cardiovascular disease, and type 2 diabetes: systematic review and meta-analysis of observational studies. BMJ 2015;351:h3978. doi:10.1136/bmj.h3978

34 Li A, Ha Y, Wang F, Li W, Li Q. Determination of thermally induced trans-fatty acids in soybean oil by attenuated total reflectance Fourier transform infrared spectroscopy and gas chromatography analysis. J Agric Food Chem 2012;60:10709-13. doi:10.1021/jf3033599

35 Goldberg T, Cai W, Peppa M, et al. Advanced glycoxidation end products in commonly consumed foods. J Am Diet Assoc 2004;104:1287-91. doi:10.1016/j.jada.2004.05.214

36 Dybing E, Sanner T. Risk assessment of acrylamide in foods. Toxicol Sci 2003;75:7-15. doi:10.1093/toxsci/kfg165

37 Peppa M, Raptis SA. Advanced glycation end products and cardiovascular disease. Curr Diabetes Rev 2008;4:92-100. doi:10.2174/157339908784220732

38 Hegab Z, Gibbons S, Neyses L, Mamas MA. Role of advanced glycation end products in cardiovascular disease. World Cardiol 2012:4:90-102 doi:10.4330/wic.v4.i4.90

39 Huang M, Jiao J, Wang J, Chen X, Zhang Y. Associations of hemoglobin biomarker levels of acrylamide and all-cause and cardiovascular disease mortality among U.S. adults: National Health and Nutrition Examination Survey 2003-2006. Environ Pollut 2018;238:852-8. doi:10.1016/j.envpol.2018.03.109

40 Semba RD, Bandinelli S, Sun K, Guralnik JM, Ferrucci L. Plasma carboxymethyl-lysine, an advanced glycation end product, and all-cause and cardiovascular disease mortality in older communitydwelling adults. J Am Geriatr Soc 2009;57:1874-80. doi:10.1111/ j.1532-5415.2009.02438x

41 Kilhovd BK, Juutilainen A, Lehto S, et al. High serum levels of advanced glycation end products predict increased coronary heart disease mortality in nondiabetic women but not in nondiabetic men: a population-based 18-year follow-up study. Arterioscler Thromb Vasc Biol 2005;25:815-20. doi:10.1161/01. ATV.0000158380.44231.fe

42 Liu ZM, Tse LA, Ho SC, et al. Dietary acrylamide exposure was associated with increased cancer mortality in Chinese elderly men and women: a 11-year prospective study of Mr. and Ms. OS Hong Kong. / Cancer Res Clin Oncol 2017;143:2317-26. doi:10.1007/ s00432-017-2477-4

43 Sutherland WH, Walker RJ, de Jong SA, van Rij AM, Phillips V, Walker HL. Reduced postprandial serum paraoxonase activity after a meal rich in used cooking fat. Arterioscler Thromb Vasc Biol 1999;19:1340-7. doi:10.1161/01.ATV.19.5.1340

44 Maiolino G, Rossitto G, Caielli P, Bisogni V, Rossi GP, Calò LA. The role of oxidized low-density lipoproteins in atherosclerosis: the myths and the facts. Mediators Inflamm 2013;2013:714653. doi:10.1155/2013/714653

45 Cook NR, Appel LJ, Whelton PK. Sodium Intake and All-Cause Mortality Over 20 Years in the Trials of Hypertension Prevention. J Am Coll Cardiol 2016;68:1609-17. doi:10.1016/j.jacc.2016.07.745

46 Larsson SC, Wolk A. Potato consumption and risk of cardiovascular disease: 2 prospective cohort studies. Am J Clin Nutr 2016;104:1245-52. doi:10.3945/ajcn.116.142422

Web appendix: Supplementary material 